\title{
C. burnetii Shedding Study In Domestic Animals in Georgia
}

\section{Marine Nikolaishvili}

Animal Disease Diagnostics Department, Laboratory of the Ministry of Agriculture, Tbilisi, Georgia

Objective

Q fever is poorly understood in Georgia and its prevalence is largely underestimated in both humans and animals. One of the main goal of the project was shedding study in domestic animals - isolation of C. burnetii from suspected seropositive animal blood, milk samples.

\section{Introduction}

Q fever is a zoonotic bacterial disease resulting from infection by Coxiella burnetii. Domestic ruminants (cattle, sheep, and goats) are considered the main reservoir for the pathogen, which can also infect humans. Q fever is poorly understood in Georgia and its prevalence is largely underestimated in both humans and animals.

In Georgia Q fever laboratory diagnostic was started and implemented at the Laboratory of the Ministry of Georgia (LMA) within GG20, ,Prevalence, Epidemiological Surveillance, and Laboratory Analysis of Coxiella burnetii in Georgia'.

\section{Methods}

LMA conducted Coxiella burnetii shedding evaluation in three specific farms from Kvemo Kartli (Tsalka, Dmanisi) and MtskhetaMtianeti (Dusheti). Seropositive cattle and small ruminants were sampled per week. Sampling lasted 7 weeks and totally 581 samples samples (blood, milk and swab) were tested. Testing were conducted in a BSL3 laboratory under BSL3 working conditions. ACCM medium was used (2XACCm-2 acidified Citrate Cysteine Medium PH- 4.75G N NaOH). The samples were incubated at $37^{\circ} \mathrm{C}$ using $\mathrm{CO}_{2}$.

\section{Results}

As a result of the study, one culture was bacteriologically isolated from seropositive cattle milk sample (the sample was taken on the third week of the study in Beshtasheni farm, Tslka, Kvemo Kartli) and confirmed by Molecular biology (PCR).

\section{Conclusions}

The study confirmed Q fever existence in Georgia. Traditionally considered an obligate intracellular agent, the requirement to be grown in tissue culture cells, embryonated eggs, or animal hosts has made it difficult to isolate $C$. Burnetii strains. Within the study one culture was isolated from the seropositive animal milk sample that was collected in the third week of the study. shedding of Coxiella burnetii in milk by infected cows appeared to be the most frequent positive sample for the bacterium. 